\title{
Kinase-catalyzed Biotinylation for Phosphoprotein Detection
}

\author{
Keith D. Green and Mary Kay H. Pflum* \\ Department of Chemistry, Wayne State University, Detroit, Michigan 48202
}

Supporting Information

\section{Materials.}

Unless otherwise stated, reagents were obtained from commercial sources and used as recommended. Trifluoroacetic acid (TFA), acetyl chloride, Acroseal methanol, and alpha-cyano-4-hydroxy cinnamic acid were purchased from Acros. Tris base, ethylenediamine tetraacetic acid (EDTA) and sodium chloride are purchased from JT Baker. $\mathrm{d}_{4}$-methanol is obtained from Cambridge Isotope Labs. Dithiothreitol (DTT), HPLC grade acetonitrile, glacial acetic acid, and glycerol were bought from Fisher Scientific. Goat anti-mouse was purchased from Southern Biotech Associates. Triton X-100 was obtained from Fluka, and ammonium bicarbonate was purchased from Sigma. Bovine serum albumin (BSA) was obtained from Santa Cruz Biotechnology.

\section{Procedures}

\section{II.A Kinase-mediated biotinylation}

II.A.1 Synthetic peptides: Each reaction contained either 200 units of PKA (New England Biolabs), 40 units of Abl kinase (New England Biolabs), or 40 units of CK2 (New England Biolabs). Either 375 pmol of kemptide peptide 2 (Promega) in the case of PKA, $150 \mathrm{pmol}$ of CK2 substrate peptide 3 (Promega), or $150 \mathrm{pmol}$ of Abl peptide substrate 4 (New England Biolabs) were also used. To create a phosphorylated peptide, 10 nmol ATP (MP Biomedicals Inc) was used. To create a biotinylated phosphopeptide, $10 \mathrm{nmol}$ ATP-biotin (Adenosine 5'triphosphate $[\gamma]$-biotinyl-3, 6, 9-trioxaundecanediamine from Affinity Labeling Technologies Inc.- CAS\# 864538-90-9) was used after evaporation of the methanol storage solvent. All reactions contained the manufacturer's specified kinase buffer and were incubated at $30^{\circ} \mathrm{C}$ for 2 hours with shaking $(1000 \mathrm{rpm})$. In the case of the reactions with CK2, better yields were obtained when $50 \mu \mathrm{M} \mathrm{MgCl}_{2}$ and $2 \%$ glycerol were added to the manufacturers recommended buffer. To analyze the products of the reactions by MALDI-TOF MS, $1 \mu \mathrm{L}$ of the crude reaction mixture was combined with $5 \mu \mathrm{L}$ of matrix (alpha-cyano-4-hydroxy cinnamic acid dissolved in $70 \%$ acetonitrile, $30 \%$ water and $0.1 \%$ TFA), evaporated on a MALDI plate, and analyzed using a Bruker Ultraflex MALDI-TOF mass spectrometer. Ion suppression is observed with biotinylated peptides, ${ }^{1}$ explaining the preponderance of peptide salts in the MS analysis (Table 1). To address the issue of biotinylated phosphopeptide stability, the Abl substrate peptide $\mathbf{5}$ was biotinylated using ATP-biotin (1b) and Abl as described and then stored at $4^{\circ} \mathrm{C}$ for several days. Using MALDI-TOF MS, no significant loss of biotin (determined by monitoring the production of unmodified phosphopeptide due to phosphoramidate hydrolysis) was observed for 3 days. Chemical biotinylation of phosphopeptides were reported previously without complications from instability. ${ }^{2}$

II.A.2 Full-length Proteins: The $\beta$-casein protein was purchased from Sigma and used in phosphorylation reactions as described in section II.B.2. To obtain full-length recombinant CREB, BL21 E. coli cells were transformed with the T7-7 CREB plasmid (a kind gift of Marc Montminy). ${ }^{3}$ The cells were grown in $50 \mathrm{~mL}$ of Luria Broth with $50 \mu \mathrm{g} / \mathrm{mL}$ ampicillin to an $\mathrm{OD}_{600}$ of 0.6 and protein expression induced with $1 \mathrm{mM}$ IPTG (Fisher Scientific). After 4 hours of protein expression, the cells were harvested at $4000 \mathrm{rpm}$ and stored as a cell pellet at $-80^{\circ} \mathrm{C}$. To lyse the cell, the cell pellet was resuspended in $1 \mathrm{~mL}$ of lysis buffer $(10 \mathrm{mM}$ Tris $\mathrm{pH} 8.0,1$ mM EDTA, $1 \mathrm{mM}$ DTT, and $1 \mathrm{mM}$ PMSF) and heated to $75^{\circ} \mathrm{C}$ for 10 minutes. The cell debris was collected by centrifugation at 12,000 rpm and the supernatant containing CREB was stored at $4^{\circ} \mathrm{C}$ and used in subsequent phosphorylation reactions without any further purification. Typically, $12.5 \mu \mathrm{L}$ of bacterial lysates were incubated with 1500 units of PKA, 20 nmol of nucleotide in 1X kinase reaction buffer. For the reactions including HeLa cell lysates (described in section II.A.3), $12.5 \mu \mathrm{L}$ of bacterial lysates containing recombinant CREB and $12.5 \mu \mathrm{L}$ of HeLa lysates were combined and mixed with $20 \mathrm{nmol}$ of nucleotide in $1 \mathrm{X}$ kinase reaction buffer either with or without 1500 units of PKA. To assess the reaction efficiency, the crude reaction products were separated using 12\% SDS-PAGE before transfer to a PVDF membrane (Immobilon $\mathrm{P}^{\mathrm{SQ}}$ - Millipore). The 
separated proteins were visualized with colloidal coomassie stain (Life Gels), ProQ Diamond Phosphoprotein Stain (Invitrogen), monoclonal anti-phosphorylated CREB S133 antibody- clone 10E9 (Upstate), or streptavidin-horseradish peroxidase conjugate (1 to 5000 dilution- Endogen) using a Storm 860 phosphoimager instrument.

II.A.3 HeLa lysates: For the reactions containing mammalian cell lysates, 20 X $10^{6} \mathrm{HeLa}$ cells obtained from the National Cell Culture Center (www.nccc.org) were lysed in $1 \mathrm{~mL}$ of lysis buffer $(50 \mathrm{mM}$ Tris pH $8,150 \mathrm{mM}$ $\mathrm{NaCl}, 10 \%$ Glycerol, $0.5 \%$ Triton X-100) containing $1 \mathrm{mM}$ PMSF (Alexis) and protease inhibitor cocktail $\mathrm{V}$ $\left(1 \mathrm{X}-\right.$ Calbiochem) by rotation at $4^{\circ} \mathrm{C}$ for 10 minutes. The cell debris was collected at $12,000 \mathrm{rpm}$ and the supernatant was stored at $4^{\circ} \mathrm{C}$ until use in subsequent phosphorylation reactions. For the reactions containing HeLa lysates alone, $25 \mu \mathrm{L}$ of lysates were combined with $20 \mathrm{nmol}$ of nucleotide in $1 \mathrm{X}$ kinase reaction buffer either with or without 1500 units of PKA and analyzed as in section II.A.2. The full gel images from the HeLa lysates labeling are shown in Figure S5.

\section{II.B Quantitative Mass Spectrometric Analysis}

II.B.1 Synthetic Peptides: Biotinylated phosphopeptides (produced with ATP-biotin) and phosphorylated peptides (produced from ATP) were generated as described in II.A.1. and the solvent was evaporated. Each peptide was incubated in a solution of $2 \mathrm{~N}$ acetyl chloride in $\mathrm{d}_{0}$-methanol (in the case of the ATP product) or $\mathrm{d}_{4^{-}}$ methanol (in the case of the ATP-biotin product) at $12^{\circ} \mathrm{C}$ for 2 hours to produce anhydrous $\mathrm{HCl}$ and afford carboxylic acid esterification, as described. ${ }^{4}$ Because the conditions of esterification were acidic, the phosphoramidate bond linking the biotin label to the peptide was cleaved, creating the phosphopeptide required for quantitative mass spectrometric analysis. In the case of PKA, which does not rely on the presence of carboxylic acids for substrate recognition, the peptides were differentially labeled prior to the kinase reaction (Scheme S1). In this case, the phosphoramidate bond cleavage required for biotin release was promoted by incubation with $50 \% \mathrm{TFA}$ at $12^{\circ} \mathrm{C}$ for 2 hours (Scheme S1). For MALDI-TOF MS, equal volumes of the isotopically differentiated phosphopeptide samples was combined and prepared as described in section II.A.1 (Figure S1, S2, and S3).

Scheme S1: Isotopic labeling for quantitative MS experiments

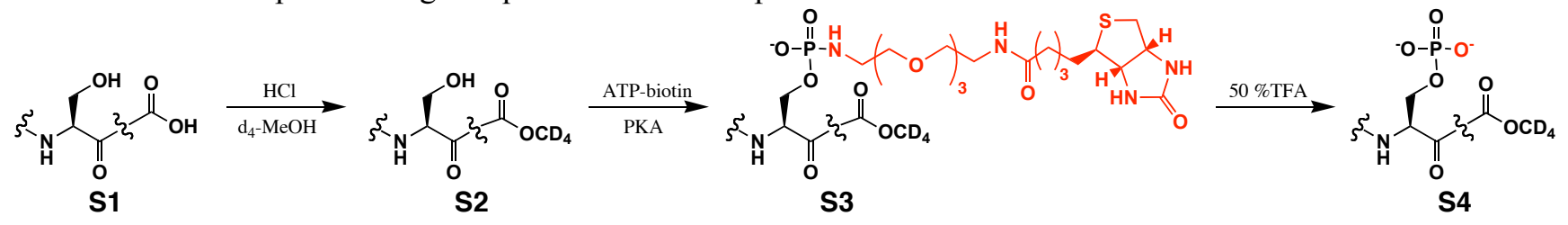

II.B.2 Full-length Proteins: $\beta$-casein $(1.8 \mathrm{nmol})$ was combined with 80 units CK2 and $20 \mathrm{nmol}$ of either ATP or ATP-biotin in the manufacturer's reaction buffer (supplemented with $50 \mathrm{mM} \mathrm{MgCl} 2$ and $2 \%$ glycerol) and incubated for 2 hours at $30^{\circ} \mathrm{C}$. The reaction was diluted with an equal volume of $50 \mathrm{mM} \mathrm{NH} \mathrm{NCO}_{3}$ before heat denaturing at $90^{\circ} \mathrm{C}$ for 60 seconds. To afford complete proteolysis, $2.25 \mu \mathrm{g}$ of sequencing grade trypsin (Promega) was incubated with the protein at $37^{\circ} \mathrm{C}$ overnight with shaking (1000rpm). The solvent was removed and the peptide fragments were subjected to acidic esterification and quantitative MS analysis (Figure S4) as described in section II.B.1.

\section{References}

1. Saurin, A. T.; Neubert, H.; Brennan, J. P.; Eaton, P., PNAS 2004, 101, (52), 17982-17987.

2. Zhou, H.; Watts, J.; Aebersold, R., Nat. Biotech. 2001, 19, (4), 375-378.

3. Parker, D.; Ferreri, K.; Nakajima, T.; LaMorte, V. J.; Evans, R.; Koerber, S. C.; Hoeger, C.; Montminy, M. R., Mol. Cell. Biol. 1996, 16, (2), 694-703.

4. Tao, W. A.; Wollscheid, B.; O'Brien, R.; Eng, J. K.; Li, X.-j.; Bodenmiller, B.; Watts, J. D.; Hood, L.; Aebersold, R., Nat. Methods 2005, 2, (8), 591-598. 


\section{Figures}

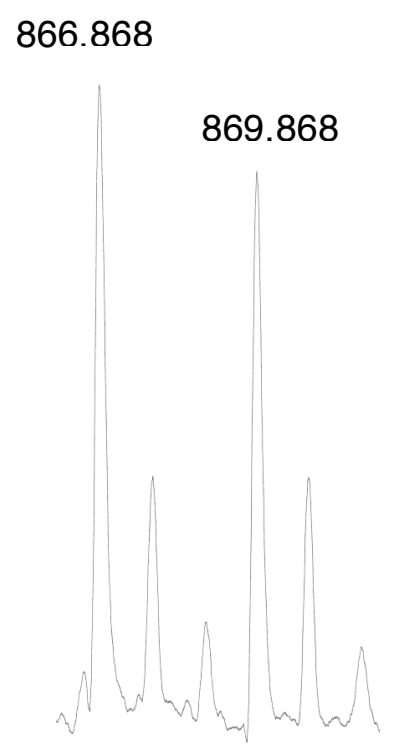

Figure S1: Quantitative MALDI analysis of kemptide (3) with PKA and ATP-Biotin (1b). The peak at 866.868 $\mathrm{m} / \mathrm{z}$ corresponds to methylated phosphopeptide due to ATP phosphorylation, while the peak at $869.868 \mathrm{~m} / \mathrm{z}$ corresponds to the deuterated phosphopeptide after phosphorylation with ATP-biotin and acidic biotin cleavage. The percent conversion in this single experiment was $78 \%$.

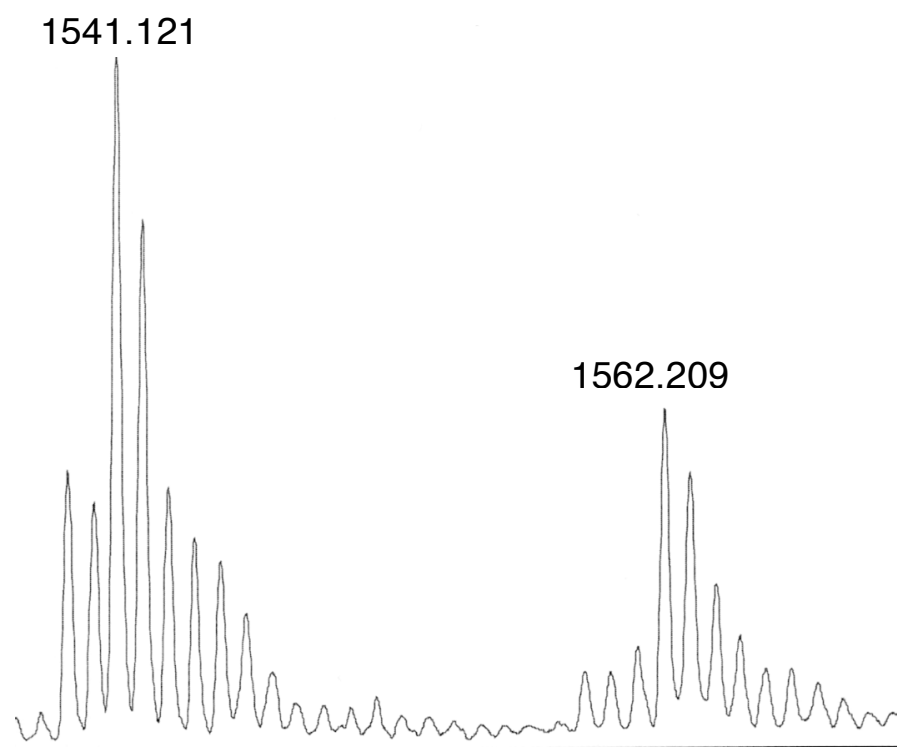

Figure S2: Quantitative MALDI analysis of CK2 peptide (4) with CK2 and ATP-Biotin (1b). The peak at $1541.121 \mathrm{~m} / \mathrm{z}$ corresponds to the hepta-methylated phosphopeptide due to ATP phosphorylation, while the peak at $1562.209 \mathrm{~m} / \mathrm{z}$ is the deuterated phosphopeptide after phosphorylation by ATP-biotin and acidic biotin cleavage. The conversion for this single experiment was 55\%. 


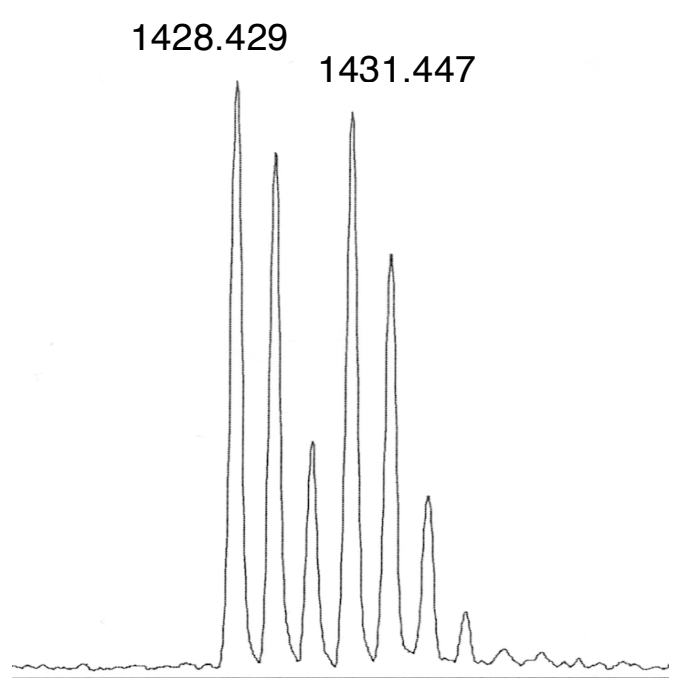

Figure S3: Quantitative MALDI analysis of Abl peptide (5) reaction with Abl and ATP-Biotin (1b). The peak at $1428.429 \mathrm{~m} / \mathrm{z}$ corresponds to the methylated phosphopeptide due to ATP phosphorylation, and the peak at $1431.447 \mathrm{~m} / \mathrm{z}$ corresponds to the deuterated phosphopeptide due to phosphorylation by ATP-biotin and acidic biotin cleavage. The conversion in this single reaction was $82 \%$.

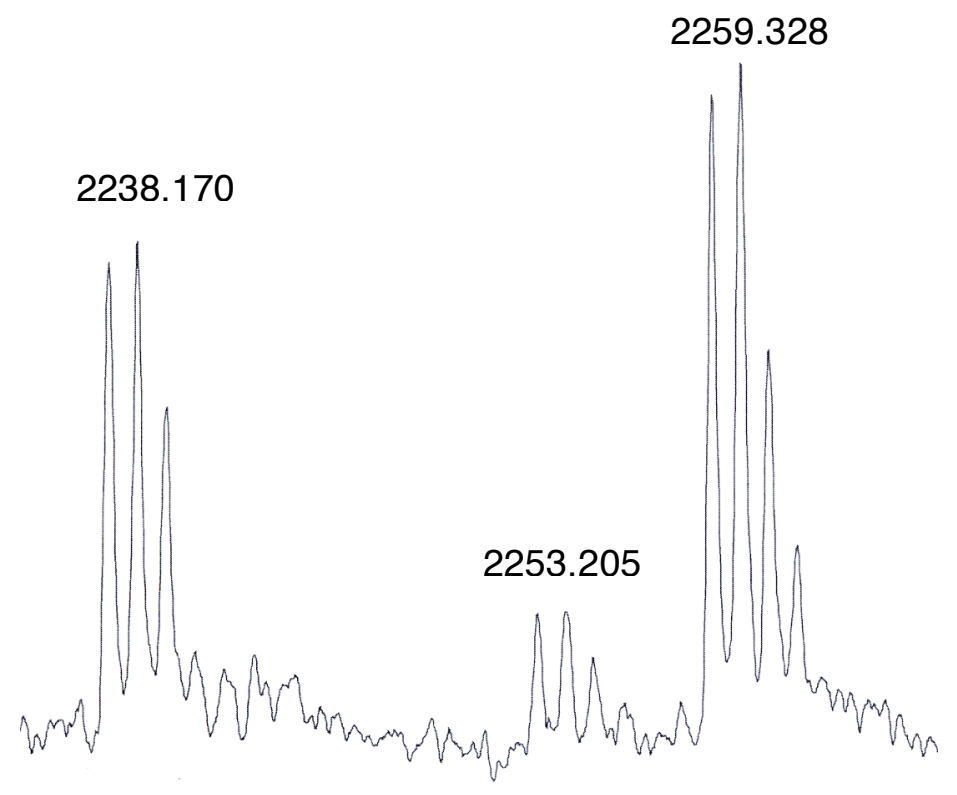

Fig S4: Quantitative MALDI analysis of trypsin digested $\beta$-casein after reaction with CK2 and either ATP or ATP-biotin (1b). The peak at $2238.170 \mathrm{~m} / \mathrm{z}$ corresponds to methylated phosphopeptide due to ATP phosphorylation, while the peak at $2259.328 \mathrm{~m} / \mathrm{z}$ corresponds to the deuterated phosphopeptide after phosphorylation by ATP-biotin and acidic biotin cleavage. The peak at $2253.204 \mathrm{~m} / \mathrm{z}$ represents a peptide present in the trypsin digest. The percent conversion in this single experiment is $73 \%$. 


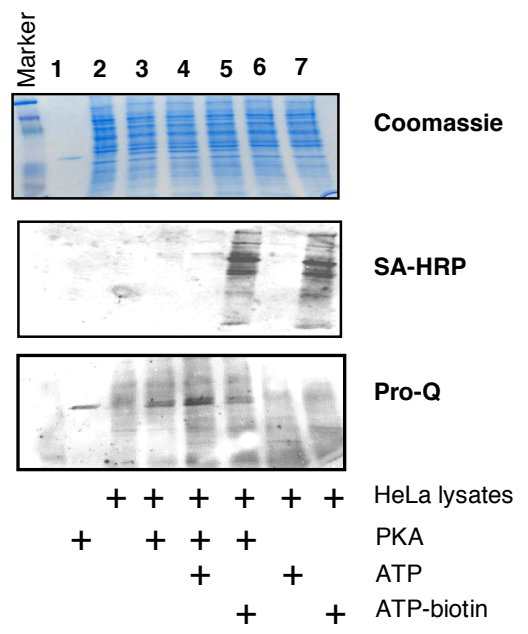

Figure S5: Full gel image of Figure 3B from the main text, including molecular weight marker. The components of each reaction are indicated below each lane. 